\title{
Altered DNA methylation profiles in blood from patients with sporadic Creutzfeldt-Jakob disease
}

\author{
Luke C. Dabin ${ }^{1}$ (1) - Fernando Guntoro ${ }^{1} \cdot$ Tracy Campbell $^{1} \cdot$ Tony Bélicard $^{2} \cdot$ Adam R. Smith $^{3} \cdot$ Rebecca G. Smith $^{3}$. \\ Rachel Raybould ${ }^{4} \cdot$ Jonathan M. Schott ${ }^{5} \cdot$ Katie Lunnon $^{3}{ }^{10} \cdot$ Peter Sarkies $^{2} \cdot$ John Collinge $^{1} \cdot$ Simon Mead $^{1}$. \\ Emmanuelle Viré ${ }^{1}[$ ]
}

Received: 10 July 2020 / Revised: 13 August 2020 / Accepted: 31 August 2020 / Published online: 12 September 2020

(c) The Author(s) 2020, corrected publication 2020

\begin{abstract}
Prion diseases are fatal and transmissible neurodegenerative disorders caused by the misfolding and aggregation of prion protein. Although recent studies have implicated epigenetic variation in common neurodegenerative disorders, no study has yet explored their role in human prion diseases. Here we profiled genome-wide blood DNA methylation in the most common human prion disease, sporadic Creutzfeldt-Jakob disease (sCJD). Our case-control study $(n=219)$, when accounting for differences in cell type composition between individuals, identified 38 probes at genome-wide significance $\left(p<1.24 \times 10^{-7}\right)$. Nine of these sites were taken forward in a replication study, performed in an independent case-control $(n=186)$ cohort using pyrosequencing. Sites in or close to FKBP5, AIM2 (2 probes), UHRF1, KCNAB2 successfully replicated. The blood-based DNA methylation signal was tissue- and disease-specific, in that the replicated probe signals were unchanged in case-control studies using SCJD frontal-cortex $(n=84)$, blood samples from patients with Alzheimer's disease, and from inherited and acquired prion diseases. Machine learning algorithms using blood DNA methylation array profiles accurately distinguished sCJD patients and controls. Finally, we identified sites whose methylation levels associated with prolonged survival in SCJD patients. Altogether, this study has identified a peripheral DNA methylation signature of SCJD with a variety of potential biomarker applications.
\end{abstract}

Keywords Prion disease $\cdot$ sCJD $\cdot$ DNA methylation $\cdot$ Blood $\cdot$ Disease duration $\cdot$ Neurodegeneration

Luke C. Dabin and Fernando Guntoro contributed equally.

Electronic supplementary material The online version of this article (https://doi.org/10.1007/s00401-020-02224-9) contains supplementary material, which is available to authorized users.

Simon Mead

s.mead@prion.ucl.ac.uk

1 MRC Prion Unit at UCL, UCL Institute of Prion Diseases, Courtauld Building, 33 Cleveland Street, London W1W 7FF, UK

2 MRC London Institute of Medical Sciences Du Cane Road London W12 0NN and Institute of Clinical Sciences, Imperial College London Du Cane Road London W12 0NN, Imperial College London, London W12 0NN, UK

\section{Introduction}

Human prion diseases are typically rapidly progressive neurodegenerative conditions associated with misfolding of prion protein $(\operatorname{PrP})[10]$. They include sporadic, Mendelian genetic, and acquired disorders which present and progress heterogeneously, although all are inevitably fatal. Neuropathologically, the diseases are characterized by spongiform changes in the grey matter with neuronal loss,

3 College of Medicine and Health, University of Exeter Medical School, Exeter University, RILD Building Level 4, Royal Devon and Exeter Hospital, Barrack Rd, Exeter EX2 5DW, UK

4 Institute of Psychological Medicine and Clinical Neurosciences, Cardiff University, UHW Main Building, Heath Park, Cardiff CF14 4XN, UK

5 Dementia Research Centre, UCL Institute of Neurology, 8-11 Queen Square, London WC1N 3AR, UK 
reactive gliosis, and the accumulation of misfolded forms of PrP. The causative and transmissible agent of prion diseases, or prion, is thought to comprise solely or predominantly of misfolded forms of PrP forming paired double helical fibrils [56]. Mechanisms inspired by the prion concept are now widely adopted in neurodegenerative diseases associated with misfolded forms of other proteins and peptides [10].

The most common form of human prion disease is sporadic CJD (sCJD), occurring almost exclusively in adults over the age of 40 years with an annual incidence of $\sim 2$ per million population. Whilst sCJD occurs seemingly at random in the population, most of the cases present between 60 and 80 years old. Prion diseases are under strong genetic control, with the most powerful risk factors being located at the PrP gene locus (PRNP), particularly the polymorphism at codon 129 in the PRNP gene where methionine $(\sim 60 \%$ allele frequency in Europeans) or valine is encoded and both homozygous genotypes are at increased risk of disease [11, 45]. Codon 129 genotype also modifies the incubation period of acquired prion diseases and the resulting clinicopathological phenotype $[12,49]$. Speed of decline in activities of daily living is fastest in those with the methionine homozygous genotype, and slowest in those with the heterozygous genotype [35, 47].

According to epidemiological case definitions, a brain biopsy or post-mortem examination is necessary to confirm a diagnosis of definite SCJD, although neurological investigations such as cerebrospinal fluid (CSF) analysis, magnetic resonance imaging (MRI), electroencephalogram (EEG) and $P R N P$ analysis can lead to a confident pre-mortem diagnosis of SCJD once it is suspected by specialist physicians. More recently, extensive research has been directed towards identification of specific and selective biomarkers such as metabolites or proteins. To date, these efforts have largely concentrated on altered protein concentrations, or the realtime quaking-induced conversion assay using CSF [59]. The need for biomarkers in easily accessible tissues such as blood is important because such tests could help make diagnoses and prognoses earlier and screen individuals before invasive procedures, tissue or blood donation. Such approaches might also improve screening of individuals for inclusions in clinical trials prior to irretrievable neuronal damage.

Epigenetic signals are emerging as biomarkers for screening and early detection of various diseases, for prognostic and treatment monitoring, and for predicting future risk of disease development [51]. Accumulating evidence suggests that epigenetic modification of gene expression regulates memory acquisition and consolidation in the healthy brain and that epigenetic dysregulation contributes to the impaired cognition and neuronal death that are associated with neurodegenerative diseases [23]. Whether these changes are causally involved in diseases remain poorly understood. However, the contribution of epigenetic mechanisms to the initial steps and disease progression in neurodegeneration is yet still poorly understood.

Here, we compared genome-wide DNA methylation profiles in whole blood taken from patients with SCJD and age-matched healthy controls and characterized the genomic distribution of differentially methylated sites and regions. We identified sites where loss of DNA methylation correlates with disease progression. We further demonstrated that the DNA methylation signature is not altered in $\mathrm{AD}$ or in other prion diseases and found that the sites affected in blood are not differentially methylated in brain. Next, we show that machine learning models trained using DNA methylation profiles can discriminate SCJD from control individuals. Finally, our findings, when used in combination with the genetic information of the patients, help refine disease duration predictions. We report the first SCJD DNA methylation-based blood signature that provides diagnostic and genotype-independent prognostic information.

\section{Methods}

\section{Patient samples and genomic DNA extraction}

Patients with definite diagnosis of sCJD according to World Health Organization criteria were recruited by the National Prion Clinic (London, UK), and other referrers in the UK between 1995 and 2018. All sCJD patients were of UK residency. Blood or DNA from control donors was sourced from Cardiff University (Cardiff, UK), or from the National Prion Clinic (London, UK). Genomic DNA was extracted from peripheral blood using either a BACC2 DNA extraction kit (GE Healthcare, IL, USA) or a Zymo Quick gDNA MiniPrep Kit (Zymo Research, CA, USA) according to the manufacturers' instructions.

GPower 3.1 [15] was used to estimate sample sizes required to power a genome-wide study. Where brain samples were used, genomic DNA was extracted from frontal cortex grey matter from 51 autopsied sCJD brains in Biosafety Level 3 facilities. 50-100 mg of tissue was transferred to a $2 \mathrm{ml}$ screw-capped tube (Eppendorf, Germany) and incubated in $450 \mu \mathrm{l}$ ATL lysis buffer (Qiagen, $\mathrm{NL}$ ) and $50 \mu \mathrm{l}$ proteinase $\mathrm{K}$ (from $20 \mathrm{mg} \mathrm{ml}^{-1}$ stock) in a Thermomixer Comfort heating block (Eppendorf, Germany) overnight at $50{ }^{\circ} \mathrm{C}$ with mixing at $800 \mathrm{rpm}$. The next day, samples were mixed with $500 \mu \mathrm{l}$ of TRIS equilibrated phenol (Sigma-Aldrich, DE) by inversion. Tubes were then centrifuged at 16,100 $\mathrm{g}$ for $5 \mathrm{~min}$ at room temperature before the upper aqueous phase was transferred to a fresh tube and lower organic phase was discarded into a phenol waste bottle. Addition of TRIS-equilibrated phenol, centrifugation and selection of the aqueous phase were repeated before $500 \mu \mathrm{l}$ of a 1:1 v/v TRIS-equilibrated phenol and chloroform 
mixture was added and mixed by inversion. Centrifugation and selection of the aqueous phase was repeated before a final addition of $500 \mu \mathrm{l}$ chloroform to the sample, which was centrifuged at $16,100 \mathrm{~g}$ for $2 \mathrm{~min}$. The upper aqueous phase was transferred to a clean tube and from containment level 3 facilities to containment level 2 facilities, where $500 \mu \mathrm{l} 100 \%$ ethanol was added and mixed to induce DNA precipitation. DNA was spooled out onto a flame-sealed glass Pasteur pipette and left to dry for $2 \mathrm{~min}$, before resuspension in a $1.5 \mathrm{ml}$ Eppendorf tube containing $500 \mu \mathrm{l}$ Tris-EDTA buffer (Sigma-Aldrich, DE). Genomic DNA from was similarly extracted from 33 non-prion control frontal cortex samples acquired from Cambridge Brain Bank (University of Cambridge, UK). Concentration of extracted DNA was measured via Qubit (Thermo Fisher, MA, USA) and integrity was assessed using gDNA Tapestation ScreenTapes (Agilent, CA, USA). Samples with a DIN $<7.0$ were excluded from the study. Ethical approval was obtained from the National Hospital Local Research Ethics Committee.

\section{Genome-wide DNA methylation profiling}

Bisulfite conversion of $500 \mathrm{ng}$ of genomic DNA was performed using the Zymo EZ-96 DNA Methylation-Gold $\mathrm{Kit}^{\mathrm{TM}}$ (Zymo, CA, USA) according to the manufacturer's instructions. All DNAs were hybridized onto the Infinium ${ }^{\circledR}$ Human Methylation $450 \mathrm{~K}$ BeadChip array (Illumina, CA, USA). Fully methylated and unmethylated DNA standards (Zymo, CA, USA) and a commercially available leukocytederived DNA standard (AMSBIO, UK) were included as comparative controls for extreme variance of global DNA methylation profiles, while control probes on the array were used to monitor bisulphite conversion efficiency. DNA methylation data have been deposited in GEO (accession number GSE156994).

\section{Identification of differentially methylated CpG loci}

Analyses were performed in RStudio version 1.0.136 using $\mathrm{R}$ v3.4.1. IDAT files were loaded into ChAMP version 2.10.2 [39] and normalised using the BMIQ method [57]. Reported values at thousands of $450 \mathrm{~K}$ array probes are known to associate with assigned positions on the BeadChip [25]. We confirmed this using singular value composition and used ComBat [26] to correct for BeadChip number and sample position batch effects. Leukocyte population heterogeneity was estimated and corrected for using the Houseman method [22]. Quantile-quantile plots and Manhattan plots were generated using an in-house script adapted from qqman version 0.1.4 [60] and the pQQ function from version 7.0.0 of the haplin package [19]. An area of $95 \%$ confidence level was shaded around the reference line in the QQ plot. For Manhattan plots, a significance threshold was drawn at Bonferroni-adjusted significance threshold of $p$ value $=1.24 \times 10^{-7}$. Principal component analysis (PCA) was performed using the $\mathrm{R}$ packages FactoMine $\mathrm{R}$ version 1.41 [30] and factoextra version 1.0.5 (https://CRAN.Rproject.org/package=factoextra). Heatmaps were produced using a script adapted from (https://github.com/obigriffith/) using either the top 38 or 1000 most significant differentially methylated $\mathrm{CpG}$ loci. Hierarchical clustering analysis was performed using the average clustering method based on Euclidean distance. Plotly (https://plot.ly/) was used to generate the pie charts showing the genomic locations of the $\mathrm{CpG}$ loci.

We used limma version 3.36.5 to build linear regression models of methylation versus disease status with age and sex included as covariates [48]. Bivariate Pearson's correlations were used to correlate identified DMPs with MRC Scale score and slope in SCJD patients, while a one-way ANOVA with Dunnett's post hoc test was used to test for association of methylation at DMPs with genotype at codon 129 of PRNP. To identify DMRs, we used the DMRcate function in ChAMP [24] setting the lambda at 500. We used Bioconductor package 'PWMEnrich' [54] to perform motif scanning and enrichment analysis across the DMPs using probe sequences extracted from the Illumina HumanMethylation450K manifest file, and on the DMRs using sequences retrieved from Bioconductor package BSgenome.Hsapiens. UCSC.hg 19 version 1.4.0 [55]. For pathways and ontology analysis, MetaCore [37] was used. Differentially methylated probes (Bonferroni corrected $p \leq 0.1$ ) and their $\Delta \beta$ values were uploaded to MetaCore and analysed as a single experiment, using the Illumina $450 \mathrm{~K}$ array background and a significance threshold of $p \leq 0.1$. Pathmap analysis was performed using Dijkstra's shortest path algorithm with a maximum node distance of 2 , using canonical pathways.

\section{Pyrosequencing}

Genomic DNA was bisulfite converted as described above. PCR primers were designed using the PyroMark ${ }^{\circledR}$ Assay Design 2.0 software (Qiagen, NL) and manually adjusted using the following criteria: (i) amplicon length less than $200 \mathrm{bp}$, (ii) sequencing read length below $40 \mathrm{nt}$, (iii) forward and reverse primers have a length of 20-25 nt and do not overlap $\mathrm{CpG}$ sites, (iv) sequencing primer does not exceed $20 \mathrm{nt}$ in length and has an optimal $\mathrm{T}^{\mathrm{m}}$ of $40^{\circ} \mathrm{C}$. Primers were checked for specificity in silico using BiSearch [2] and optimum annealing temperatures were determined using PCR with an annealing temperature gradient between 52 and $62{ }^{\circ} \mathrm{C}$. PCR amplification of bisulfite-converted DNA was performed in a Veriti 96-Well Thermo Cycler (Thermofisher, MA, USA) using the following mastermix per $1 \mu \mathrm{l}$ bisulifteconverted DNA: 1X Buffer B1 (Solis BioDyne, EE), $25 \mathrm{mM}$ $\mathrm{MgCl}_{2}$ (Solis BioDyne, EE), $10 \mathrm{mM}$ dNTP mix (Promega, 
WI, USA), $10 \mu \mathrm{M}$ forward/reverse primer mix (Supplementary Table 5, online resource), 1 U HotFire Polymase (Solis BioDyne, EE) and RNAse-free water (Thermofisher, MA, USA) to a total reaction volume of $20 \mu \mathrm{l}$. PCR products were sequenced using the Pyromark ${ }^{\mathrm{TM}} \mathrm{Q} 96$ system (Qiagen, NL) according to the manufacturer's protocol. Where the assay permitted, a non-CpG cytosine was selected as a control for complete bisulfite conversion. Statistical analysis was performed in SPSS version 25 using a linear regression model of methylation (\%) versus disease status, with age and sex as covariates. Pyrosequencing data are presented as Tukey box plots, where the box is divided by the median and extends across the interquartile range. Whiskers protrude from the box up to 1.5 times the interquartile range, and outlying values are plotted as single points beyond them.

\section{Cortisol profiling}

$500 \mu \mathrm{l}$ aliquots of sera taken from patients and controls between 10 a.m. and 4 p.m. were sent to The Doctor's Laboratory (Sonic Healthcare Ltd., London, UK), where cortisol concentration was measured using the Elecsys Cortisol II assay (Roche: 11875116160) on a Cobase 801 module. Difference in cortisol concentration between SCJD and control donors was calculated in SPSS version 25 using a linear regression model with age and sex as covariates.

\section{Machine learning classification}

Preprocessing of the $\beta$ values from the 1000 most significantly altered loci from $105 \mathrm{sCJD}$ and 105 control patients was performed in $\mathrm{R}$ version 3.5.1 and Rstudio version 1.1.456. The deep learning neural network model was created using the machine learning Tensorflow version 1.12.0 library [34] (Abadi et al., 2015) run in Python 3 platform. The dataset was randomized and partitioned into training and test sets in a 50:50 ratio. The architecture of the model consisted of three dense hidden layers using the rectified linear unit activation function, and a sigmoid output layer to compute a score between 0 and 1 for classifying SCJD status. Furthermore, L2 weight regularization of $1 \times 10^{-3}$ was applied to the hidden layers to impose a penalty to the cost function and reduce overfitting. The model was compiled using the Adam optimizer [13] with a learning rate of $5 \times 10^{-5}$. Classification model performance was measured using two different metrics: accuracy and binary crossentropy loss. The model was then fit into the training and test sets in 400 epochs with a batch size of 8 . The model was compared to a basic random forest classifier imported from version 0.21 of the Python 'sklearn' library [46], which was also used to analyse the ROC curve, AUC and 10-k fold validation.

\section{Kaplan-Meier curves}

Survival curves were generated using the packages ggplot2 and survminer [28] using the ggsurvplot() function. Survival curves were plotted based on data from 102 sCJD patients after removing 12 patients without complete clinical information. For each of the 38 DMPs, beta values from each patient were categorized into "High" or "Low" according to the mean methylation levels. The confidence interval and $p$ values were computed using default options (log-rank test for $p$ value). For the Codon129 MM-MV-VV comparisons, a threshold $p$ value $<0.05$ was used so that there is a significant difference within the particular subgroup, i.e. methylation level has an effect. For the effect of DNA methylation on survival independent of codon 129, "High versus Low", $p$ value $>0.05$ was used. For the combined survival analysis, beta values from the DMPs were transformed into $z$-scores after passing parametric test (Shapiro-Wilk cg01084918 $p$ value $=0.4967 ; \operatorname{cg} 05343106 p$ value $=0.4372 ; \operatorname{cg} 17641710$ $p$ value $=0.2549$ ). The average $z$-score is then used to inform levels of methylation ( $z$-score $>0$, "High"; $z$-score $<0$, "Low").

\section{Results}

\section{DNA methylation profiles are altered in blood from SCJD patients}

We profiled DNA methylation in whole blood from UK sCJD $(n=114)$ patients and age- and sex-matched controls ( $n=105$; Table 1) using Illumina Infinium HumanMethylation450 BeadChip arrays (450 $\mathrm{K}$ arrays). One sample and 82,158 probes were discarded, which either failed quality control or were included in published blacklists [39, 43] (Supplementary Fig. 1a, online resource). To identify an association between methylation $\beta$ values [4] and SCJD disease status, we used a mixed linear regression model, including age and sex as covariates. Principal component analysis (PCA) of pre-processed data showed that the first two components were highly correlated with the contrasted groups, accounting for $9.7 \%$ and $6.7 \%$ of the variability, respectively (Fig. 1a and Supplementary Fig. 1b, online resource), demonstrating that stringent quality control and adjustment steps removed sources of variation from factors unrelated to the biological variables. In this initial analysis, uncorrected for cell composition, we found 22,398 differentially methylated positions (DMPs) between healthy controls and SCJD patients (Bonferroni correction; $p<1.24 \times 10^{-7}$ ) (Fig. 1b). Of those, 283 sites showed an absolute change in methylation greater than $10 \%(\Delta \beta>|0.1|)$ in sCJD (Fig. 1b and Supplementary Table 1, online resource). 
Table 1 Sample characteristics of the individuals included in the analyses

\begin{tabular}{llrllll}
\hline Study stage & Group & $N$ & Average age (range) & Sex $(\% \mathrm{~F})$ & $\begin{array}{l}\text { Codon 129 }(\%) \\
\text { MM:MV:VV }\end{array}$ & $\begin{array}{l}\text { Average MRC } \\
\text { Scale score } \\
\text { (range) }\end{array}$ \\
\hline Exploratory & sCJD & 114 & $67.6(49-85)$ & 50.9 & $46: 22: 32$ & $6.3(0-20)$ \\
& Control & 106 & $69.3(41-83)$ & 55.7 & $44: 43: 13$ & 20 \\
Replication & sCJD & 72 & $67.3(26-86)$ & 58.3 & $54: 23: 23$ & $4.5(0-18)$ \\
& Control & 114 & $78.2(61-93)$ & 64.9 & Unknown & 20 \\
Specificity & Control & 114 & $78.2(61-93)$ & 64.9 & Unknown & 20 \\
& AD & 60 & $72.8(70-77)$ & 56.7 & Unknown & Unknown \\
& iCJD & 18 & $46.4(41-53)$ & 11.1 & $27: 73: 00$ & $10.3(0-18)$ \\
& IPD & 11 & $46.0(39-68)$ & 45.4 & $64: 36: 00$ & $17.0(8-20)$ \\
Brain & sCJD & 51 & $59.2(38-87)$ & 41.2 & $65: 10: 25$ & 0 \\
& Control & 33 & $74.0(41-89)$ & 54.2 & Unknown & 0 \\
\hline
\end{tabular}

$s C J D$ sporadic CJD, $A D$ Alzheimer's disease, $i C J D$ iatrogenic CJD, IPD inherited prion disease
Given that whole blood is a heterogeneous collection of different cell types, each with their own distinct DNA methylation landscape, we next used Houseman's statistical method to estimate the relative proportions of cell type components [22]. Figure 1 shows that cell proportion estimates (the sum of which is forced to $100 \%$ ) differed subtly between samples in the study. The algorithm estimated that granulocytes comprised more than $75 \%$ of the blood cell types in 58 sCJD patients and 16 healthy controls, which is the upper limit of the normal range for granulocytes proportion in whole blood. When accounting for differences in the six cell types, the number of differentially methylated probes that passed genome-wide significance $(p<1.24 \times 10-7)$ dropped from 22,398 to 38 (Supplementary Fig. 1c-e, online resource and Table 2). Strikingly, this cell type correction substantially reduced the inflation factor $(\lambda)$ of our epigenome-wide analysis from 5.12 to 1.72 (Fig. 1d). Hierarchical clustering analysis (Pearson minus one correlation) of the significant 38 DMPs from the 219 patients and controls identified 3 clusters of sCJD cases and one cluster of controls (Supplementary Fig. 1f), raising the possibility of heterogeneity in DNA methylation associated with the disease. In contrast to disease status, PRNP codon 129 genotype and sex of the patients did not cluster within the data. Of the significant 38 DMPs, 4 were hypomethylated with a mean effect size of $\Delta \beta-0.037\left(95 \% \mathrm{CI} \pm 2.01 \times 10^{-2}\right)$ and 34 were hypermethylated with a mean effect size of $\Delta \beta$ $+0.022\left(95 \% \mathrm{CI} \pm 3.67 \times 10^{-3}\right)$. Figure 1 shows a Manhattan plot for these DMPs. Altogether, these results suggest SCJD patients have distinct blood DNA methylation profiles compared with healthy controls.

\section{Characteristics of differentially methylated sites in SCJD}

A circular ideogram was used to visualize the chromosome distribution of the 38 positions (DMPs) identified in SCJD patients' blood (Fig. 2a and Supplementary Table 2, online resource, respectively). Genomic features of the 38 DMPs and 67 differentially methylated regions (DMRs) were compared to the null distribution of $\mathrm{CpG}$ probes included in the array. No significant associations between features and DMPs were observed, while as expected DMRs had a significant overlap with Transcription Start Sites (DMPs: Chi-square $=3.0267, \mathrm{df}=6, p$ value $=0.8055$; DMRs: Chi-squared $=204.64, \mathrm{df}=6, p$ value $<2.2 \times 10^{-16}$ ) (Supplementary Fig. 2a, online resource). Next, we asked if sCJD-specific DMPs were enriched for transcription factor binding motifs. Using PWMEnrich [54], we identified the most significantly over-represented motifs within the DMPs (Fig. 2b and Table 3) as those recognized by GLTPD1, a negative regulator of interleukin-1 beta secretion $\left(p\right.$ value $\left.=1.55 \times 10^{-5}\right)$. Other transcription factors identified included cell cycle regulators $D B P$ and $D I A B L O$, an inhibitor of apoptosis protein (IAP)-binding protein ( $p$ value $=2.63 \times 10^{-5}$ and $p$ value $=0.0002$, respectively). To further investigate potential consequences of the site-specific DNA methylation in sporadic CJD, we curated a list of RefSeq genes overlapping each of the 38 differentially methylated site and performed downstream analysis using Metacore Gene Ontology [37]. The results in Fig. 2c show enrichment of negative regulators of protein tyrosine phosphatase activity, negative regulators of adenylate cyclaseactivating adrenergic receptors, and negative regulators of 

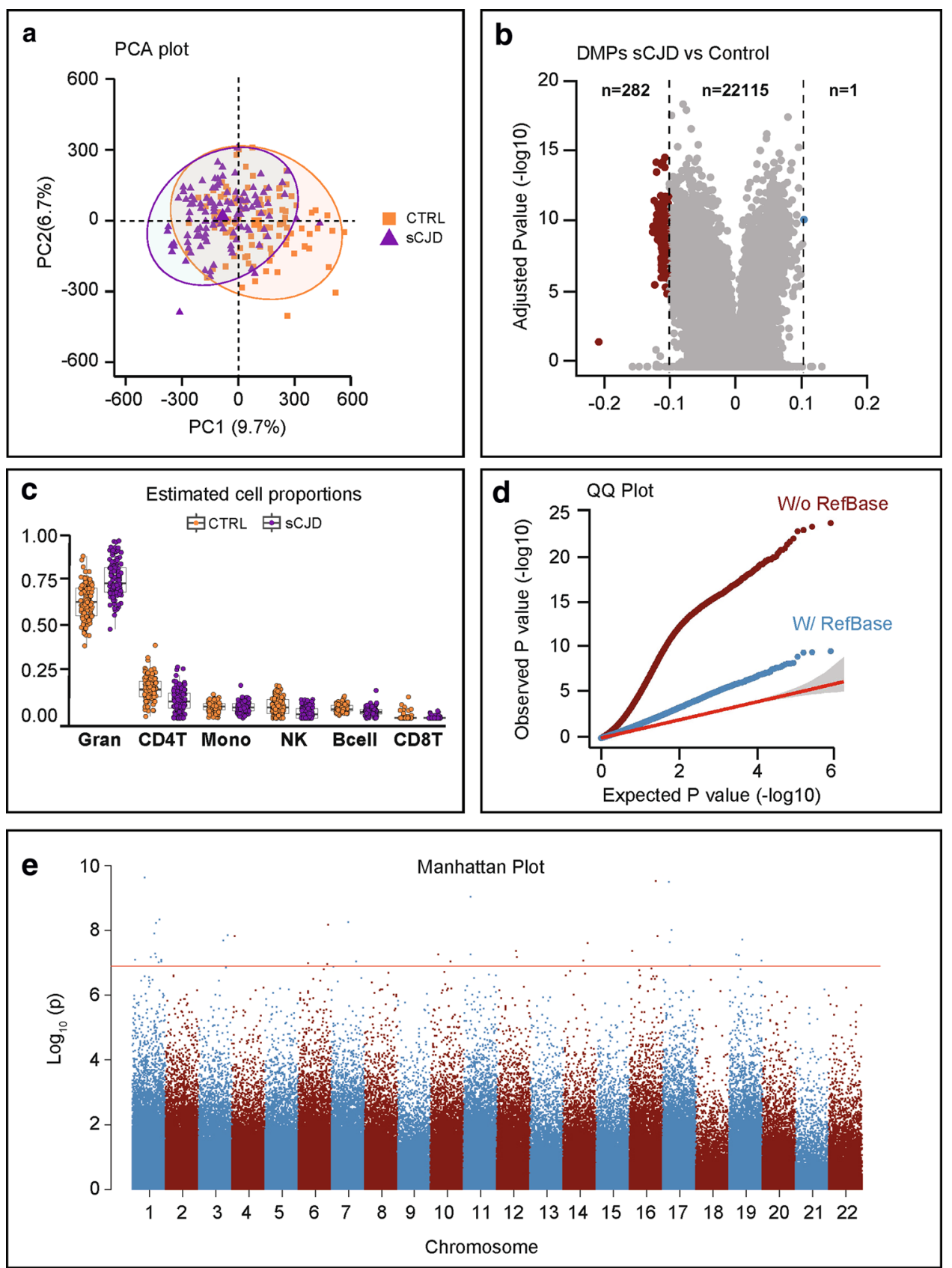

Fig. 1 Genome-wide differential methylation in sporadic CJD blood. a Principal component analysis (PCA) of 219 DNA methylation profiles showing the first (PC1) and second (PC2) principal components (9.7\% and $6.7 \%$ of the total variance). $95 \%$ confidence ellipses are drawn around the two groups: sCJD (triangles, purple) and healthy controls (squares, orange). b Volcano plot DMP association analysis ( $\mathrm{X}$ and $\mathrm{Y}$ chromosomes were excluded from analysis), corrected for sex but not for blood cell composition. $X$-axis represents (effect size) adjusted mean delta difference, $Y$-axis represents $-\log 10$ ( $q$ value). Vertical lines indicate delta beta $>|0.1|$. c Tukey box plots showing proportions of six different cell types as estimated by Houseman algorithm in SCJD (purple) and healthy controls (orange). Wil-
coxon-Mann-Whitney test to identify differences between SCJD and control: granulocytes $p=1.47 \mathrm{e}-14$; CD4T $p=5.05 \mathrm{e}-11$; monocytes $p=0.78$; natural killers cells $p=1.10 \mathrm{e}-08$; B cell $p=1.55 \mathrm{e}-10$; CD8T $p=0.59$. d Quantile-quantile plots (QQ plots) of the distribution of observed $-\log 10$ association $p$ values against the expected null distribution without (dark red) and with (blue) cell type correction. The red line represents the expected distribution with $95 \%$ confidence interval. e Manhattan plot of probes associated with disease status corrected for blood cell type composition. Red line indicates significance threshold (Bonferroni-adjusted $=1.24 \times 10^{-7}$ ). $X$-axis represents ranked chromosomes, $Y$-axis represents $-\log 10$ ( $p$ value) 
Table 2 List of 38 differentially methylated positions

\begin{tabular}{|c|c|c|c|c|c|c|}
\hline & Cpg loci & Delta $\beta$ & Adjusted $p$ value & Chromosome & Gene & Feature \\
\hline 1 & $\operatorname{cg} 10636246$ & -0.0405317 & $9.58 \times 10^{-5}$ & 1 & AIM2 & TSS 1500 \\
\hline 2 & $\operatorname{cg} 02481950$ & 0.01975019 & 0.00012239 & 16 & METTL9 & Body \\
\hline 3 & $\operatorname{cg} 14427590$ & 0.02273577 & 0.0001344 & 17 & & IGR \\
\hline 4 & $\operatorname{cg} 05740793$ & 0.04394973 & 0.0003859 & 11 & & IGR \\
\hline 5 & $\operatorname{cg} 13965201$ & 0.0290582 & 0.00193489 & 1 & & IGR \\
\hline 6 & $\operatorname{cg} 21540367$ & 0.00991642 & 0.0023237 & 7 & LRCH4 & Body \\
\hline 7 & $\operatorname{cg} 05001044$ & 0.05331962 & 0.00236357 & 1 & MIR1977 & TSS 1500 \\
\hline 8 & $\operatorname{cg} 09048334$ & 0.03476521 & 0.00272688 & 6 & & IGR \\
\hline 9 & $\operatorname{cg} 22519265$ & 0.01080344 & 0.00394136 & 17 & ATP2A3 & 3'UTR \\
\hline 10 & $\operatorname{cg} 02448796$ & 0.03283323 & 0.00510789 & 1 & KCNAB2 & Body \\
\hline 11 & $\operatorname{cg} 17641710$ & 0.0238572 & 0.00582785 & 3 & GNAI2 & Body \\
\hline 12 & $\operatorname{cg} 03819286$ & 0.02679297 & 0.00622737 & 16 & MGRN1 & TSS 1500 \\
\hline 13 & $\operatorname{cg} 10855342$ & 0.00640701 & 0.00634387 & 4 & ALPK1 & $5^{\prime} \mathrm{UTR}$ \\
\hline 14 & $\operatorname{cg} 15197458$ & 0.01401478 & 0.00792343 & 19 & & IGR \\
\hline 15 & $\operatorname{cg} 00832928$ & 0.02884309 & 0.00838196 & 3 & SELT & Body \\
\hline 16 & $\operatorname{cg} 22688566$ & 0.02832814 & 0.00925244 & 17 & MYO18A & Body \\
\hline 17 & $\operatorname{cg} 25966751$ & 0.02254565 & 0.01015631 & 14 & & IGR \\
\hline 18 & $\operatorname{cg} 20056593$ & 0.01654389 & 0.01723034 & 12 & & IGR \\
\hline 19 & cg27229664 & 0.02206902 & 0.01770546 & 16 & KIAA0513 & $5^{\prime} \mathrm{UTR}$ \\
\hline 20 & $\operatorname{cg} 22505006$ & 0.02903491 & 0.02133475 & 1 & ZBTB7B & $5^{\prime} \mathrm{UTR}$ \\
\hline 21 & $\operatorname{cg} 05343106$ & 0.02028 & 0.0229423 & 11 & DNAJB13 & TSS200 \\
\hline 22 & $\operatorname{cg} 17714703$ & 0.03310906 & 0.02311756 & 19 & UHRF1 & Body \\
\hline 23 & $\operatorname{cg} 07081759$ & 0.0249997 & 0.02342596 & 10 & FAM53B & Body \\
\hline 24 & $\operatorname{cg} 13444131$ & 0.00917468 & 0.0237334 & 19 & DYRK1B & $5^{\prime} \mathrm{UTR}$ \\
\hline 25 & $\operatorname{cg} 22434506$ & 0.00961761 & 0.02800703 & 12 & IFFO1 & Body \\
\hline 26 & $\operatorname{cg} 17515347$ & -0.0473728 & 0.02813301 & 1 & AIM2 & TSS 1500 \\
\hline 27 & cg20003976 & 0.01435695 & 0.02817662 & 1 & ACADM & TSS 1500 \\
\hline 28 & $\operatorname{cg} 09007354$ & 0.0237598 & 0.03272807 & 1 & GLIS1 & $5^{\prime} \mathrm{UTR}$ \\
\hline 29 & $\operatorname{cg} 20285559$ & 0.01014608 & 0.03348693 & 1 & THAP3 & Body \\
\hline 30 & $\operatorname{cg} 19769147$ & 0.01949328 & 0.03474175 & 14 & PACS2 & Body \\
\hline 31 & $\operatorname{cg} 24843003$ & 0.02944661 & 0.03518602 & 19 & DAZAP1 & Body \\
\hline 32 & $\operatorname{cg} 03393322$ & 0.0084795 & 0.03783743 & 7 & SDK1 & Body \\
\hline 33 & $\operatorname{cg} 04757081$ & 0.01403053 & 0.03821293 & 10 & & IGR \\
\hline 34 & $\operatorname{cg} 01084918$ & 0.02041746 & 0.03843211 & 1 & FAM40A & TSS 1500 \\
\hline 35 & $\operatorname{cg} 01101459$ & 0.02894185 & 0.04051284 & 1 & & IGR \\
\hline 36 & $\operatorname{cg} 03546163$ & -0.0535485 & 0.04329215 & 6 & FKBP5 & $5^{\prime}$ UTR \\
\hline 37 & $\operatorname{cg} 21393135$ & 0.00327746 & 0.04491255 & 6 & VARS & Body \\
\hline 38 & $\operatorname{cg} 21155515$ & -0.0075201 & 0.04995584 & 17 & CANT1 & 1stExon \\
\hline
\end{tabular}

TSS 1500 located 200-1500 nucleotides upstream transcriptional start site, TSS 200 located 0-200 nucleotides upstream transcriptional start site, Body body of the gene, IGR intergenic regions, 5'UTR and 3'UTR are, respectively, $5^{\prime}$ and $3^{\prime}$ untranslated regions
cAMP-mediated signalling (Supplementary Fig. 2b, online resource).

Using these 38 probes, we set out to examine whether patient metadata would help identify disease-modifying loci. We investigated the effect of PRNP codon 129 polymorphism (which is known to alter susceptibility to prion disease and rate of disease progression) [35], age at onset and disease duration (binned in three groups: less than 100 days, longer than 200 days, or between 100 and 200 days) and found no significant difference between any of these groups (Supplementary Fig. 3a-c, online resource). Next, we explored disease severity using the MRC Scale, which rates functional impairment in SCJD from a score of 20 (healthy) to 0 (moribund) [58]. We correlated the patient's score measured at the time each sample was collected with the methylation values at each DMP. The test was performed genome-wide (Supplementary Fig. 3d, online resource) and again with the 38 significant DMPs only (Fig. 2d). Figure 2 

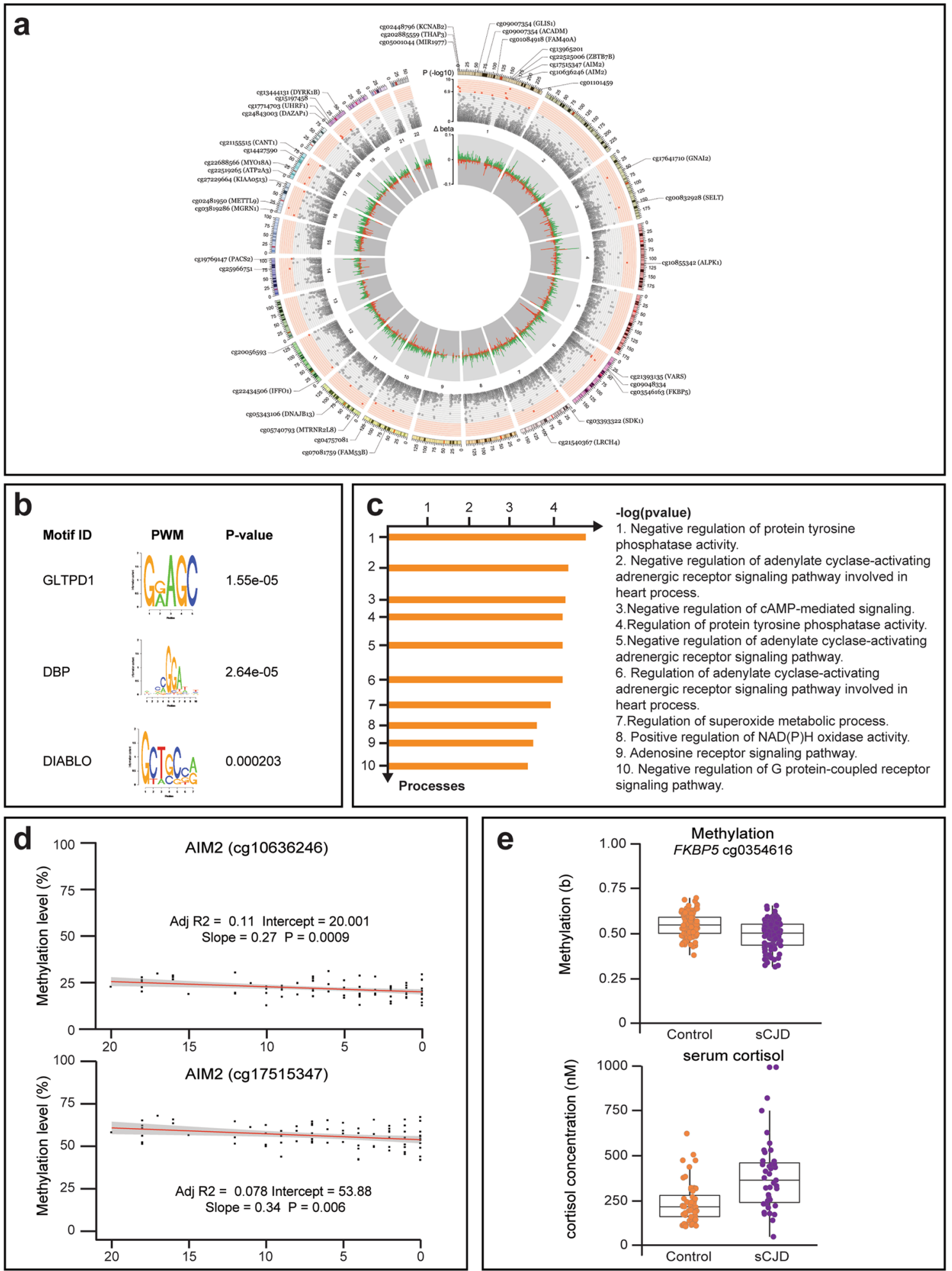

shows that methylation at two probes located in the promoter of AIM2 (identified as a hit locus in the case-control study) decreased with disease progression ( $\operatorname{cg} 10636246$ : slope $=0.27, p$ value $=0.0013 ; \operatorname{cg} 17515347$ slope $=0.32$, $p$ value $=0.012$, whilst none of the other probes tested showed significant association (Supplementary Fig. 3e, online resource).

Given the previously reported link between FKBP5 and neurodegeneration [5], our finding that the promoter of FKBP5 is demethylated in sCJD (Fig. 2e and Table 2) 
४Fig. 2 Key findings replicated using pyrosequencing in an independent case-control cohort. a Circos plot of epigenome-wide methylation levels in SCJD. Outermost circle represents the chromosome ideogram. Middle circle shows $p$ values $(-\log 10)$ of the top 25,000 most significant DMPs (Bonferroni significance threshold of $p<1.24 \times 10-7$ or $-\log 10(p)>6.9$ is distinguished with a red background). Significant DMPs are shown in red and labelled accordingly. The innermost circle represents the $\Delta \beta$ values across the genome, with hypermethylation in green and hypomethylation in red. b Top ten motifs enriched in sequences within \pm 122 bases flanking the CG found under 'Forward_Sequence' heading in the Illumina HumanMethylation450 manifest file of the 38 significant DMPs. Ranking based on $p$ value. $\mathbf{c}$ Top ten gene ontologies enriched in genes overlapping DMPs as identified using MetaCore ( $p$ value threshold=0.1). d Pearson coefficients between MRC Scale score with hypomethylation at two CpGs in the AIM2 promoter (cg10636246 and cg17515347). e Left: DNA methylation levels (\%) at FKBP5 cg03546163 from the discovery study in SCJD patients $(n=114$; purple) and healthy controls ( $n=105$; orange) limma $p=1.07 \mathrm{e}-07$, corrected $p=0.043$. Right: serum cortisol concentrations (nM) in 39 sCJD patients (purple, $239.8 \mathrm{nM}$ ) and 52 controls (orange, $387.6 \mathrm{nM}$ ) $\left(p=6.6 \times 10^{-5}\right)$

prompted us to investigate the cortisol levels in sCJD patients. FKBP5 acts as a cochaperone in modulating glucocorticoid receptor activity in the brain and periphery $[62,64]$. We analysed cortisol levels in sera from 39 sCJD patients and 52 healthy controls. Figure 2 shows that cortisol levels were significantly elevated in sera from sCJD patients, with median cortisol concentration being $147.9 \mathrm{nM}$ higher in sCJD patients $\left(95 \%\right.$ CI 77.8-218.1 nM, $p$ value $\left.=6.6 \times 10^{-5}\right)$.

\section{The identified DNA methylation signature is unique to blood and SCJD}

Next, we aimed at replicating these findings and explore the disease and tissue specificity of the associations. To this end, we designed a second case/control study and determined the sample size required to power individual bisulfite pyrosequencing assays at each DMPs. An independent cohort of 72 sCJD and 114 age-matched controls was recruited (Table 1), and DNA methylation at candidate sites was profiled using pyrosequencing. Out of the 38 DMPs, 7 probes at 6 loci were selected for replication based on Bonferroni-adjusted statistical significance, effect size, and association with clinical metrics (MRC Scale score) observed in the discovery study (at or close to AIM2 [cg10636246 and $\operatorname{cg} 17515347]$, FKBP5, METTL9, UHRF1, KCNAB2, MIR1977). Two more sites were used as controls: one within the prion gene (PRNP) and another within $A N K 1$, a gene whose hypermethylation has consistently been reported in Alzheimer's disease (AD) [33]. DNA methylation levels at these two control sites remained unchanged in the discovery study. In total, seven sites identified from the discovery study replicated (Fig. 3a): five DMPs and two controls sites. Given the length of the amplicon analysed via the pyrosequencing assays, this allowed us to quantify another five $\mathrm{CpG}$ sites not present on the $450 \mathrm{~K}$ array, adjacent to the tested DMPs in FKBP5, AIM2, and UHRF1, which also exhibited significant differential methylation between SCJD and healthy controls (Fig. 3b). Altogether, the replication study identified a sporadic CJD methylation signature that comprises a total of ten sites (5 DMPs from the array and 5 sites from the pyrosequencing) overlapping four genes (AIM2, FKBP5, METTL9, UHRF1).

Next, we wanted to establish the disease specificity of the DNA methylation signature in the context of the differential diagnosis of dementia. Blood samples from Alzheimer's disease, iatrogenic CJD, and inherited prion disease patients were collected, and pyrosequencing was used to measure DNA methylation at the ten sites (Supplementary Table 3, online resource). Figure 3 shows that none of the ten sites showed significantly altered DNA methylation levels in any of the tested non-sCJD cohorts, suggesting the DNA methylation signature is specific to SCJD.

We investigated whether changes observed in SCJD blood reflected DNA methylation alterations in the brain. The same pyrosequencing assays were performed using frontal cortex-derived DNA obtained from 51 sCJD patients and 33 non-cognitively impaired controls. Intriguingly, none of the sCJD-specific sites differentially methylated in blood were differentially methylated in brain (Fig. 3d). Altogether, these results demonstrate that a DNA methylation signature identified in blood from sCJD patients replicates in an independent case-control cohort using a different technology and that the signal is not found in SCJD brain, or in the blood of other prion disease or Alzheimer's disease patients.

\section{DNA methylation array profiles to refine SCJD diagnosis and disease duration}

Next, we sought to investigate whether DNA methylation changes could identify potential avenues for SCJD patient management by acting as diagnostic or prognostic biomarkers. To explore if DNA methylation array profiles could discriminate sCJD from healthy controls, we applied a deep learning neural network classifier to our data. For each individual, the 1000 most significantly altered sites identified from the discovery study (114 sCJD and 105 controls) were selected and partitioned into training and test sets (50:50 ratio, with equal proportions of controls and patients in each set). Model accuracy varied during training, with an overall positive trend across sequential epochs. After 200 epochs, validation accuracy appeared to plateau and the model started to overfit (Supplementary Fig. 3a, b, online resource). The model performed well in minimizing loss also called "binary cross-entropy loss", indicating that the predicted probability converged to the actual label. After tenfold cross-validation, the model had an accuracy of 87.24 $(95 \% \mathrm{CI} \pm 3.16 \%)$ with an upper limit of $91.43 \%$ accuracy. 
Table 3 PMWEnrich motifs

\begin{tabular}{lllll}
\hline Rank & Target & id & Raw Score & $p$ value \\
\hline 1 & GLTPD1 & MGC10334 & 1.927 & $1.55 \mathrm{e}-05$ \\
2 & DBP & M5338_1.02 & 1.37 & $2.63 \mathrm{e}-05$ \\
3 & UW.Motif.0555 & UW.Motif.0555 & 7.02 & 0.0001 \\
4 & DIABLO & DIABLO & 2.46 & 0.0002 \\
5 & EBF1 & M5364_1.02 & 1.43 & 0.0002 \\
6 & ATF3 & M4683_1.02 & 5.54 & 0.0002 \\
7 & UW.Motif.0654 & UW.Motif.0654 & 5.43 & 0.0002 \\
8 & UW.Motif.0283 & UW.Motif.0283 & 15.02 & 0.0002 \\
9 & CERS4 & LASS4 & 1.34 & 0.0003 \\
10 & GOT1 & GOT1 & 1.14 & 0.0003 \\
\hline
\end{tabular}

Top ten most significant position weight matrix (PWM) motifs found enriched in the 38 DMPs. The first column is the rank, the second shows the target name, and the next column is the motif ID. This ID comes from the MotifDb package and can be used to look up further information about the motif. The fifth column gives the estimated $p$ value

As shown in Fig. 4, the receiver operating characteristic (ROC) curve, the trained neural network model demonstrated a better performance compared to a basic random forest classifier with an AUC of 0.979 compared to 0.885, respectively (sensitivity 0.91 ; specificity 0.80 ). When trained using only the 38 significantly altered loci identified in the discovery study, the model achieved an accuracy of $79.45 \%$ $( \pm 1.09 \%)$.

Finally, we evaluated the association between DNA methylation levels and survival. In our cohort, as expected, codon 129 of the prion gene PRNP strongly impacts disease duration: MV heterozygous individuals had the longest disease duration, whilst MM homozygous carriers die soonest (Supplementary Fig. 3c, online resource). To evaluate the influence of DNA methylation on survival, DNA methylation levels at each of the 38 DMPs were divided into high or low, based on the mean levels and correlated with disease duration. We found that elevated methylation levels at eight DMPs [cg01084918 (FAM40A), cg05343106 (DNAJB13), cg09007354 (GLISI), cg13965201, cg17641710 (GNAI2), cg17714703 (UHRF1), cg25966751 and cg27229664 (KIAA0513)] were associated with a longer survival in patients with SCJD (Supplementary Fig. 3d, online resource). When taking the genotype of PRNP codon 129 into account, this analysis revealed that the level of DNA methylation at two sites (UHRF1 and KIAA0513) refines the prediction on disease duration for MM patients only (Fig. 4b). When not accounting for the effect of codon 129, thee DMPs had an effect on survival: $\operatorname{cg} 05343106$ (DNAJB13), cg17641710 (GNAI2), and cg17714703 (UHRF1). This effect was seen in the "Low" methylation group of cg05343106 (DNAJB13) and $\operatorname{cg} 17641710$ (GNAI2), and "High" methylation group of cg17714703 (UHRF1). Furthermore, combining together these three loci methylation profiles provided greater accuracy than each locus in predicting clinical outcomes. The distribution of beta values from these three DMPs was not significantly different from normality, thus we transformed them into $z$-scores (Shapiro-Wilk $\operatorname{cg} 01084918 p$ value $=0.4967 ; \operatorname{cg} 05343106 p$ value $=0.4372 ; \operatorname{cg} 17641710$ $p$ value $=0.2549)$. Average $z$-scores were correlated ( $z$-score $>0$, "High"; $z$-score $<0$, "Low") with disease duration (Supplementary Table 4, online resource). Figure 4 shows that SCJD patients with higher levels of DNA methylation levels at those three sites had a 97 days longer median survival time, independently of PRNP genotype at codon 129. Together, these results demonstrate a potential utility of profiling DNA methylation in whole blood from patients with SCJD: these profiles can help discriminate sCJD patients from sex- and age-matched healthy controls and may help predict disease duration.

\section{Discussion}

Whilst DNA methylation has become increasingly studied in the context of neurodegenerative disorders, DNA methylation profiles have not yet been investigated in human prion diseases. Here, we performed a case-control study to analyse the relationship between DNA methylation and sporadic CJD using 405 peripheral blood samples from patients and controls. The discovery study used a genome-wide $450 \mathrm{~K}$ Illumina BeadChip array, with replication using a second technology, pyrosequencing. Seven of nine sites that we identified successfully replicated. We went on to show that these effects were tissue and disease specific and could be exploited for diagnostic and biomarker purposes. Overall, we highlight the potential of DNA methylation array profiling of peripheral blood for a rare and serious neurodegenerative disorder.

Like GWAS, the more recently developed EWAS is subject to biases, in particular variability in the measured methylome differences between case and controls groups unrelated to the pathobiology of interest. Strategies have been developed to measure and correct for biases, in particular we found Houseman's reference-based algorithm that corrects for alterations in cell composition proved successful in reducing the study-wide inflation [22, 61]. In this case, observed differences in the methylome might have resulted from a change in the cell types in blood that contributed to DNA in the study. The Housman algorithm estimates the relative proportion of major cell types in blood samples, using validated methylation markers. No differences in SCJD blood cell types have previously been detected $[8$, 31], although to date no large-scale studies have explored this aspect. The residual inflation of our discovery study (1.72) is higher than the lambda observed in similar studies 
[29] and raises the possibility that the false positive rate is not entirely controlled. In blood, comorbidity with systemic diseases and environmental factors such as nutrition [17] and environmental chemicals [21] have been linked to inflation in EWAS.

We questioned the relevance of the magnitude of the effect size we report here compared with the effects seen in cancerous tissues [50]. The mild to moderate effect size observed in SCJD blood could be caused by the fact that the signal is observed in the periphery rather than in the main tissue affected by the disease. To date, no genomewide DNA methylation profiling has been reported in brain tissues in sporadic CJD. However, similarly mild effect sizes (around 10\%) have been reported for significant DNA methylation change observed in brain samples from other neurodegenerative disorders such as Alzheimer's disease (AD) [1], multiple system atrophy (MSA) [3], and amyotrophic lateral sclerosis (ALS) [18]. Another possible explanation for the mild effect size is that the difference in DNA methylation is cell type specific, and therefore the signal is diluted in whole blood. If this was the case, changes in DNA methylation at identified sites should be evaluated in different blood fractions. In line with this possibility is the fact that effect sizes are systematically greater in our replication study, where results were not corrected for cell type composition. Similarly, whether these DNA methylation alterations become magnified over the course of disease remains to be investigated, as we did not test patients longitudinally. We replicated our findings in an independent case-control cohort of blood samples using an independent technology, namely pyrosequencing. Furthermore, differentially methylated sites in sporadic CJD patients remained unaffected in blood from other neurodegenerative disorders (iatrogenic CJD, inherited prion diseases, Alzheimer's disease). One possible explanation for this is the fact that SCJD patients are generally at an advanced neurological state at diagnosis when blood samples taken compared to other prion diseases and AD. To date, it remains difficult to confidently identify loci that replicate across studies given the few, relatively small EWAS studies in neurodegenerative diseases, their differing designs and number of samples analysed [16].

Concordance of DNA methylation signatures between blood and brain has been reported in ALS [18]. However, the vast majority of the literature suggests that the degree of cross-tissue correlation for DNA methylation signals is not very high [6]. Studies in AD, Parkinson's disease (PD), and Huntington Disease (HD) all show very little overlap between blood and brain DNA methylation signals $[6,14$, 20]. Our findings that DNA methylation profiles in peripheral blood do not mirror those in the frontal cortex in SCJD corroborate these reports. Of note, these samples did not belong to the same individuals. Whether this general lack of correlation is due to the nature of the samples that are being compared (blood samples are taken from living individuals whilst brain samples are collected post-mortem) requires further investigation.

Although blood-based DNA methylation might not be generally considered as a surrogate for brain tissues, DNA methylation profiles detected in peripheral tissues might remain useful as biomarkers [16]. Our findings support this. First, we report that loss of DNA methylation at two sites on the AIM2 promoter correlates with disease severity. AIM2 is a key component of the inflammasome pathway, a component of the innate immune system that drives the production of the inflammatory cytokine interleukin-1 $\beta$ (IL-1 $\beta$ ) in response to microbial and nonmicrobial signals [52]. In yeast, AIM2 triggering induces a prion-like polymerization of ASC into filaments that provide platforms for activating inflammatory cytokine production [7, 32]. However, prion pathogenesis does not seem to lead to inflammasome activation in mice [44]. Second, we show that SCJD patients display a concomitant decrease in FKBP5 DNA methylation and elevated cortisol levels. FKBP5 binds to glucocorticoid receptors and modulates glucocorticoid sensitivity. Epigenetic regulation of $F K B P 5$ and its consequences on patient's behaviour is well documented: accelerated agerelated decreases in FKBP5 methylation are associated with childhood trauma and depressive phenotypes [63], whilst increased DNA methylation levels of FKBP5 have been found in patients suffering from post-traumatic stress disorders (PTSD) and major depressive syndromes [27]. Such changes are reminiscent of the alterations observed in SCJD. Although additional functional work is needed to clarify the relationship between sCJD, FKBP5 and the hypothalamic pituitary adrenal (HPA) axis (where $F K B P 5$ plays a major role), it could be that the HPA axis provides a link between the pathology in the brain and the periphery.

Several lines of evidence support the implication of $F K B P 5$ in prion diseases. Work from the Soto group showed that FK506, a calcineurin $(\mathrm{CaN})$ inhibitor which binds to $F K B P 5$, substantially decreased the severity of clinical signs in mice presenting symptoms of prion disease. In the same study, the authors report that FK506, also known as tacrolimus, reduces brain degeneration and increases survival [40]. Another study by Nakagaki et al. demonstrated that FK506 markedly reduced the abnormal form of prion protein in the cell cultures [42]. Stocki et al. proposed that FK506 treatment results in a profound reduction in $\operatorname{PrP}^{\mathrm{C}}$ expression due to a defect in the translocation of $\operatorname{PrP}^{\mathrm{C}}$ into the endoplasmic reticulum with subsequent degradation by the proteasome [53]. More recently, treatment with FK506 suppressed typical SCJD pathology (gliosis) and significantly prolonged the survival of sCJD-inoculated mice [41]. Finally, FKBP5 DNA methylation decreases along the life span; this age-related decrease is not confounded by blood cell type heterogeneity and occurs in purified immune cell subtypes [63]. The 

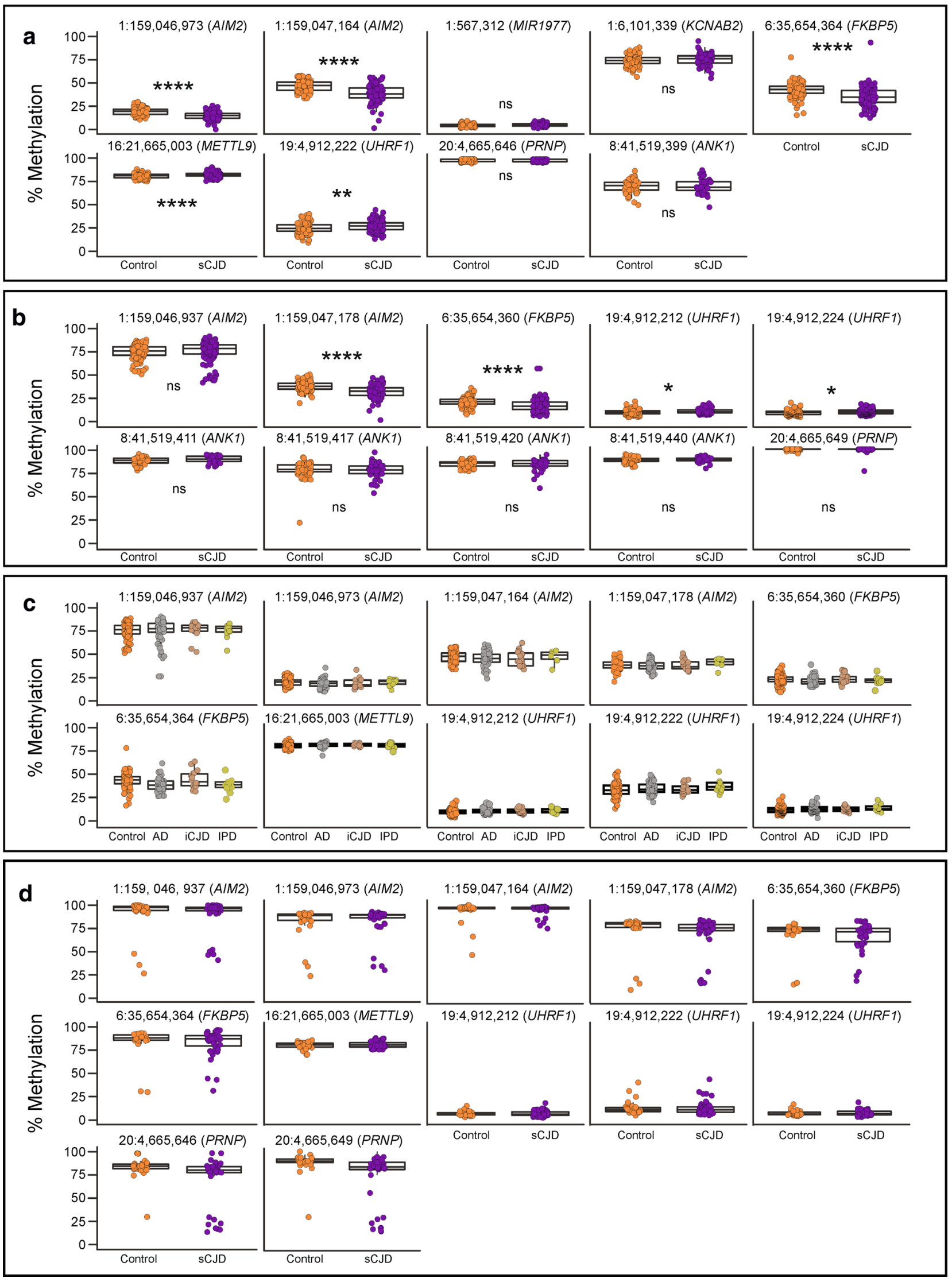
4 Fig. 3 Differential methylation signature is unique to SCJD and to blood. a DNA methylation levels (\%) at each DMPs chosen for replication by pyrosequencing SCJD patients (purple) and controls (orange). Labels above each plot show genomic coordinates and overlapping genes. b DNA methylation levels (\%) at $\mathrm{CpG}$ sites adjacent to DMPs in SCJD patients (purple) and controls (orange). Labels above each plot show genomic coordinates and overlapping genes. c DNA methylation (\%) at replicated DMPs in Alzheimer's disease (grey), iatrogenic CJD (brown) and inherited prion disease patients (green) compared to controls (orange). d Methylation at replicated sites in frontal cortex-derived DNA from SCJD patients (purple) and non-demented controls (orange). See Supplementary Table 3, online resource, for all $p$ values. $p$ value $<0.05(*) ; p$ value $<0.01(* *) ; p$ value $<0.001(* * *) ; p$ value $<0.0001(* * * *)$

same group also showed that FKBP5 upregulation promotes NFkB-related peripheral inflammation and chemotaxis. The role played by FKBP5 in inflammation and PTSD resonates with the function of another gene found to be differentially methylated in SCJD blood: AIM2. Indeed, PTSD cases are more likely to have high levels of C-reactive protein (CRP), a widely used measure of peripheral inflammation, and this association is mediated by methylation at the AIM2 locus [38].

We acknowledge several limitations of this study. Having independent replication and a larger sample size of DNA methylation data would further increase the generalizability of the classifiers identified in this study. Additionally, since methylation values can change throughout the life span, it will be insightful to evaluate the methylation signature over the course of the disease, longitudinally. In the long-term, we seek to determine when the alterations to DNA methylation patterns begin to show relative to the onset of prion diseases, and whether robust and stable DNA methylation patterns can predict the onset of disease in those at high risk of the disorder. We also aim at investigating DNA methylation profiles in first-degree relatives of the SCJD patients. We were underpowered to confidently determine whether some changes discovered in SCJD were shared with other very rare prion diseases, so it remains possible our findings associate with multiple aetiological groups. Further, whilst we compared to non-prion disease neurodegenerative disorders, these affect more specific brain functions in early stages, and are less aggressive in progression. These superficial diseaserelated differences may contribute to observed differences in blood DNA methylation, not necessarily only pathways specific to prion disease pathobiology. Finally, the brain samples were not selected because of PrP abnormalities, yet all cases had misfolded PrP immunoreactivity confirmed on neuropathological examination.

What are the functional consequences of these mild changes for cellular malfunction and disease? Future work will help investigate the mechanisms that underlie role of the selected $\mathrm{CpG}$ on sCJD establishment and progression. In line with this, our study can only provide correlative evidence for a blood-based sCJD-specific DNA methylation signature that robustly discriminates SCJD patients from controls, and other types of prion disease and AD patients. EWAS studies do not allow us to infer whether the DNA methylation observed represents a cause or consequence of sporadic CJD. Further investigations and functional studies will be required to understand better the contribution of epigenetic changes to sCJD.

Finally, our results suggest that DNA methylation profiling could be of use to refine SCJD disease management. We show that a data-driven machine learning approach using DNA methylation profiles accurately distinguishes sCJD patients from healthy individuals using peripheral blood. To date, our study is the first to report an assay that is capable of identifying SCJD patients from a blood sample, and that discriminates between sCJD and AD. Moreover, it is also the first study to suggest that DNA methylation could be used as a blood biomarker in human prion diseases. In line with this, we additionally demonstrated that a risk score based on DNA methylation of three identified sites predicts disease duration. Work from Zhang et al. recently demonstrated that DNA methylation age acceleration associated with ALS survival [65]. The finding that DNA methylation levels on two sites (KIAA0513 and UHRF1) refined survival information driven by $P R N P$ codon 129 genotype is informative, particularly for individuals most at risk. PRNP codon 129 methionine homozygosity is associated with shorter disease duration in SCJD [36] and therefore combining genetic and epigenetic information provides further insights than genotype only. None of these sites overlapped with established ageing-related CpGs. Future work in independent cohorts of sCJD patients is needed before these methods might be considered for clinical use.

Regardless of the underlying mechanisms, our results demonstrate that non-protein-mediated information about sCJD disease status is present in blood and suggest that mapping such DNA methylation patterns alterations, with future independent replication, might be of use for testing and counselling. Future work will unravel whether DNA methylation is also altered in acquired prion diseases, which 
a

a ROC curve
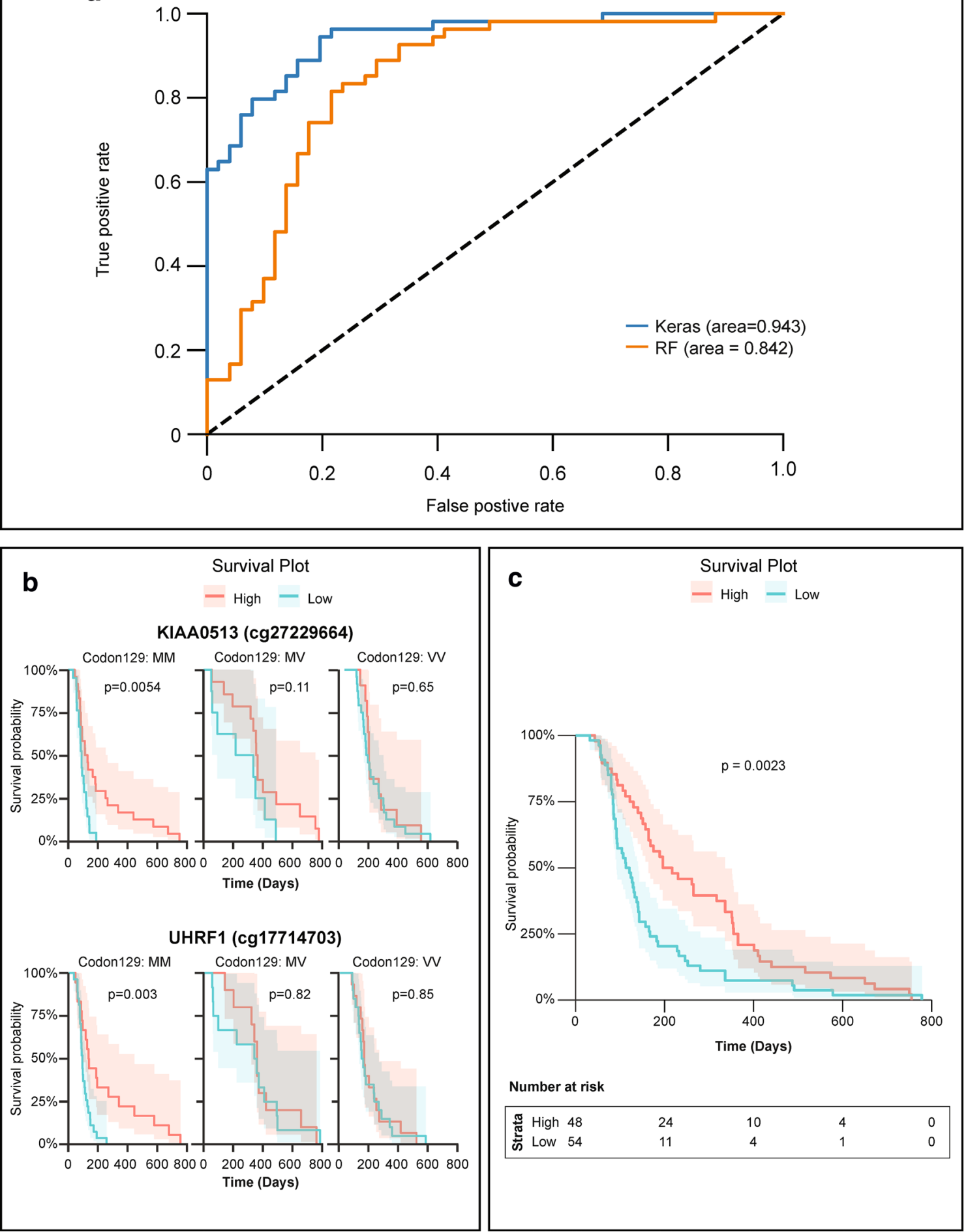

Fig. 4 Diagnostic and prognostic utility of a DNA methylation in sCJD. a Receiver operating characteristic (ROC) curve performance comparison between neural network model (Keras; blue) and random forest classifier (RF; orange) on the validation set. b Kaplan-Meier survival analysis. Patients were divided into three groups based on the genotype at PRNP codon 129 (MM, VV or MV). Patients were divided into high (above median; red) and low (below media; green)

DNA methylation values at KIAA0513 and UHRF1 DMPs. $p$ values were calculated using the log-rank test. c Survival analysis for three sites (DNAJB13, GNAI2, UHRF1) independent of PRNP genotype. Beta values from these three DMPs were transformed into $z$-scores. Kaplan-Meier curves using the average $z$-score $(z$-score $>0$, "High"; $z$-score $<0$, "Low"). See Supplementary Table 4, online resource, for median survival values 
involve peripheral pathogenesis. This study is meaningful in providing new avenues for understanding sporadic CJD disease mechanisms and identifying biomarkers which complement existing clinical signals in the periphery.

Acknowledgements We are extremely grateful to Prof Sebastian Brandner and Dr Zane Jaunmuktane for their continued support and advices. We thank all our colleagues and patients who have participated in the National Prion Monitoring Cohort Study and provided blood samples since 2008. We would like to acknowledge the NHS National Prion Clinic Team and Joanna Field for assistance with collation of patient metadata; Gaganjit Madhan Kaur and Mark Kristiansen from UCL Genomics for performing the array hybridisation. We wish to also thank Ankur Chakravarthy, Rebecca Smith, and Prof Stephan Beck for helpful advice and technical assistance. We are very grateful to Richard Newton for his help with figures and graphics. The study was funded by the Medical Research Council (UK), Alzheimer's Research UK, the NIHR Queen Square Dementia Biomedical Research Unit and the NIHR Biomedical Research Centre at University College Hospitals NHS Foundation Trust. We are very grateful to patients and volunteers who have donated blood samples and their time to research and have made this study possible. LD is supported by a Leonard Wolfson Foundation PhD fellowship. FG is supported by a NIHR Department of Health grant.

Author contributions LD, SM and EV designed experiments. LD, FG and TC performed experiments. LD, FG, SM and EV interpreted data. SM and JC assessed patients and clinical parameters. LD and FG conducted bioinformatic and statistical analyses. TB, PS, AS, RS and KL provided technical support; RR and JMS provided samples. LD, SM, and EV wrote the manuscript. All authors approved the manuscript.

\section{Compliance with ethical standards}

Conflict of interest Prof Collinge is a director and shareholder of DGen Limited (London), an academic spinout company working in the field of prion disease diagnosis, decontamination, and therapeutics. None of the other authors report any conflict of interest.

Open Access This article is licensed under a Creative Commons Attribution 4.0 International License, which permits use, sharing, adaptation, distribution and reproduction in any medium or format, as long as you give appropriate credit to the original author(s) and the source, provide a link to the Creative Commons licence, and indicate if changes were made. The images or other third party material in this article are included in the article's Creative Commons licence, unless indicated otherwise in a credit line to the material. If material is not included in the article's Creative Commons licence and your intended use is not permitted by statutory regulation or exceeds the permitted use, you will need to obtain permission directly from the copyright holder. To view a copy of this licence, visit http://creativecommons.org/licenses/by/4.0/.

\section{References}

1. Abadi M (2015) TensorFlow: large-scale machine learning on heterogeneous distributed systems. arXiv. arXiv:1603.04467

2. Altuna M, Urdanoz-Casado A, Sanchez-Ruiz de Gordoa J, Zelaya MV, Labarga A, Lepesant JMJ et al (2019) DNA methylation signature of human hippocampus in Alzheimer's disease is linked to neurogenesis. Clin Epigenet 11:91. https://doi.org/10.1186/s1314 8-019-0672-7

3. Aranyi T, Varadi A, Simon I, Tusnady GE (2006) The BiSearch web server. BMC Bioinform 7:431. https://doi. org/10.1186/1471-2105-7-431

4. Bettencourt C, Foti SC, Miki Y, Botia J, Chatterjee A, Warner TT et al (2020) White matter DNA methylation profiling reveals deregulation of HIP1, LMAN2, MOBP, and other loci in multiple system atrophy. Acta Neuropathol 139:135-156. https://doi. org/10.1007/s00401-019-02074-0

5. Bibikova M, Barnes B, Tsan C, Ho V, Klotzle B et al (2011) High density DNA methylation array with single $\mathrm{CpG}$ site resolution. Genomics 98:288-295. https://doi.org/10.1016/j.ygeno .2011 .07 .007

6. Blair LJ, Nordhues BA, Hill SE, Scaglione KM, O'Leary JC 3rd, Fontaine SN et al (2013) Accelerated neurodegeneration through chaperone-mediated oligomerization of tau. J Clin Invest 123:4158-4169. https://doi.org/10.1172/JCI69003

7. Braun PR, Han S, Hing B, Nagahama Y, Gaul LN, Heinzman JT et al (2019) Genome-wide DNA methylation comparison between live human brain and peripheral tissues within individuals. Transl Psychiatry 9:47. https://doi.org/10.1038/s41398-019-0376-y

8. Cai X, Chen J, Xu H, Liu S, Jiang QX, Halfmann R et al (2014) Prion-like polymerization underlies signal transduction in antiviral immune defense and inflammasome activation. Cell 156:12071222. https://doi.org/10.1016/j.cell.2014.01.063

9. Choi EM, Geschwind MD, Deering C, Pomeroy K, Kuo A, Miller BL et al (2009) Prion proteins in subpopulations of white blood cells from patients with sporadic Creutzfeldt-Jakob disease. Lab Invest 89:624-635. https://doi.org/10.1038/labin vest. 2009.30

10. Cohen J (1969) Statistical power analysis for the behavioral sciences. Academic Press, New York

11. Collinge J (2016) Mammalian prions and their wider relevance in neurodegenerative diseases. Nature 539:217-226. https://doi. org/10.1038/nature20415

12. Collinge J, Poulter M, Davis MB, Baraitser M, Owen F, Crow TJ et al (1991) Presymptomatic detection or exclusion of prion protein gene defects in families with inherited prion diseases. Am J Hum Genet 49:1351-1354

13. Collinge J, Whitfield J, McKintosh E, Beck J, Mead S, Thomas DJ et al (2006) Kuru in the 21st century-an acquired human prion disease with very long incubation periods. Lancet 367:2068-2074. https://doi.org/10.1016/S0140-6736(06)68930-7

14. Edgar RD, Jones MJ, Meaney MJ, Turecki G, Kobor MS (2017) BECon: a tool for interpreting DNA methylation findings from blood in the context of brain. Transl Psychiatry 7:e1187. https:// doi.org/10.1038/tp.2017.171

15. Faul F, Erdfelder E, Buchner A, Lang AG (2009) Statistical power analyses using $\mathrm{G}^{*}$ Power 3.1: tests for correlation and regression analyses. Behav Res Methods 41:1149-1160. https://doi. org/10.3758/BRM.41.4.1149

16. Fransquet PD, Lacaze P, Saffery R, McNeil J, Woods R, Ryan J (2018) Blood DNA methylation as a potential biomarker of dementia: a systematic review. Alzheimers Dement 14:81-103. https://doi.org/10.1016/j.jalz.2017.10.002

17. Friso S, Choi SW (2387S) Gene-nutrient interactions and DNA methylation. J Nutr 132:2382S-2387S. https://doi.org/10.1093/ $\mathrm{jn} / 132.8 .2382 \mathrm{~S}$

18. Gijselinck I, Van Mossevelde S, van der Zee J, Sieben A, Engelborghs S, De Bleecker J et al (2016) The C9orf72 repeat size correlates with onset age of disease, DNA methylation and transcriptional downregulation of the promoter. Mol Psychiatry 21:1112-1124. https://doi.org/10.1038/mp.2015.159 
19. Gjessing HK, Lie RT (2006) Case-parent triads: estimating single- and double-dose effects of fetal and maternal disease gene haplotypes. Ann Hum Genet 70:382-396. https://doi.org/10.111 1/j.1529-8817.2005.00218.x

20. Hannon E, Lunnon K, Schalkwyk L, Mill J (2015) Interindividual methylomic variation across blood, cortex, and cerebellum: implications for epigenetic studies of neurological and neuropsychiatric phenotypes. Epigenetics 10:1024-1032. https://doi. org/10.1080/15592294.2015.1100786

21. Hou L, Zhang X, Wang D, Baccarelli A (2012) Environmental chemical exposures and human epigenetics. Int J Epidemiol 41:79-105. https://doi.org/10.1093/ije/dyr154

22. Houseman EA, Molitor J, Marsit CJ (2014) Reference-free cell mixture adjustments in analysis of DNA methylation data. Bioinformatics 30:1431-1439. https://doi.org/10.1093/bioinformatics/ btu029

23. Hwang JY, Aromolaran KA, Zukin RS (2017) The emerging field of epigenetics in neurodegeneration and neuroprotection. Nat Rev Neurosci 18:347-361. https://doi.org/10.1038/nrn.2017.46

24. Jaffe AE, Murakami P, Lee H, Leek JT, Fallin MD, Feinberg AP et al (2012) Bump hunting to identify differentially methylated regions in epigenetic epidemiology studies. Int J Epidemiol 41:200-209. https://doi.org/10.1093/ije/dyr238

25. Jiao C, Zhang C, Dai R, Xia Y, Wang K, Giase G et al (2018) Positional effects revealed in Illumina methylation array and the impact on analysis. Epigenomics 10:643-659. https://doi. org/10.2217/epi-2017-0105

26. Johnson WE, Li C, Rabinovic A (2007) Adjusting batch effects in microarray expression data using empirical Bayes methods. Biostatistics 8:118-127. https://doi.org/10.1093/biostatistics/kxj037

27. Kang HJ, Yoon S, Lee S, Choi K, Seol S, Park S et al (2020) FKBP5-associated miRNA signature as a putative biomarker for PTSD in recently traumatized individuals. Sci Rep 10:3353. https://doi.org/10.1038/s41598-020-60334-6

28. Kassambara A, Kosinski M, Biecek P, Fabian S (2017) survminer: drawing survival curves using'ggplot2'. R package version 03

29. Kingma DP, Ba J (2014) Adam: a method for stochastic optimization. arXivorg. arXiv:1412.6980

30. Lardenoije R, Roubroeks JAY, Pishva E, Leber M, Wagner H, Iatrou A et al (2019) Alzheimer's disease-associated (hydroxy) methylomic changes in the brain and blood. Clin Epigenetics 11:164. https://doi.org/10.1186/s13148-019-0755-5

31. Lê S, Josse J, Husson F (2008) FactoMineR: an R package for multivariate analysis. J Stat Softw. https://doi.org/10.18637/jss. v025.i01

32. Lee ST, Roh JK (2009) Innocent white blood cells in sporadic Creutzfeldt-Jakob disease? Lab Invest 89:612-613. https://doi. org/10.1038/labinvest.2009.42

33. Lu A, Wu H (2015) Structural mechanisms of inflammasome assembly. FEBS J 282:435-444. https://doi.org/10.1111/ febs. 13133

34. Lunnon K, Smith R, Hannon E, De Jager PL, Srivastava G, Volta $\mathrm{M}$ et al (2014) Methylomic profiling implicates cortical deregulation of ANK1 in Alzheimer's disease. Nat Neurosci 17:1164 1170. https://doi.org/10.1038/nn.3782

35. Mead S, Mahal SP, Beck J, Campbell T, Farrall M, Fisher E, Collinge J (2001) Sporadic-but not variant-Creutzfeldt-Jakob disease is associated with polymorphisms upstream of PRNP exon 1. Am J Hum Genet 69:1225-1235. https://doi.org/10.1086/324710

36. Mead S, Burnell M, Lowe J, Thompson A, Lukic A, Porter MC et al (2016) Clinical trial simulations based on genetic stratification and the natural history of a functional outcome measure in Creutzfeldt-Jakob disease. JAMA Neurol 73:447-455. https://doi. org/10.1001/jamaneurol.2015.4885
37. Metacore. https://support.clarivate.com/LifeSciences/s/artic le/Metacore-How-to-cite-Metacore-in-publications?langu age $=$ en_US

38. Miller MW, Maniates H, Wolf EJ, Logue MW, Schichman SA, Stone A et al (2018) CRP polymorphisms and DNA methylation of the AIM2 gene influence associations between trauma exposure, PTSD, and C-reactive protein. Brain Behav Immun 67:194-202. https://doi.org/10.1016/j.bbi.2017.08.022

39. Morris TJ, Butcher LM, Feber A, Teschendorff AE, Chakravarthy AR, Wojdacz TK et al (2014) ChAMP: 450k chip analysis methylation pipeline. Bioinformatics 30:428-430. https://doi. org/10.1093/bioinformatics/btt684

40. Mukherjee A, Morales-Scheihing D, Gonzalez-Romero D, Green K, Taglialatela G, Soto C (2010) Calcineurin inhibition at the clinical phase of prion disease reduces neurodegeneration, improves behavioral alterations and increases animal survival. PLoS Pathog 6:e1001138. https://doi.org/10.1371/journal.ppat.1001138

41. Nakagaki T, Satoh K, Ishibashi D, Fuse T, Sano K, Kamatari YO et al (2013) FK506 reduces abnormal prion protein through the activation of autolysosomal degradation and prolongs survival in prion-infected mice. Autophagy 9:1386-1394. https://doi. org/10.4161/auto.25381

42. Nakagaki T, Ishibashi D, Mori T, Miyazaki Y, Takatsuki H, Tange $\mathrm{H}$ et al (2020) Administration of FK506 from late stage of disease prolongs survival of human prion-inoculated mice. Neurotherapeutics. https://doi.org/10.1007/s13311-020-00870-1

43. Nordlund J, Backlin CL, Wahlberg P, Busche S, Berglund EC, Eloranta ML et al (2013) Genome-wide signatures of differential DNA methylation in pediatric acute lymphoblastic leukemia. Genome Biol 14:r105. https://doi.org/10.1186/gb-2013-14-9-r105

44. Nuvolone M, Sorce S, Schwarz P, Aguzzi A (2015) Prion pathogenesis in the absence of NLRP3/ASC inflammasomes. PLoS ONE 10:e0117208. https://doi.org/10.1371/journal.pone.0117208

45. Palmer MS, Dryden AJ, Hughes JT, Collinge J (1991) Homozygous prion protein genotype predisposes to sporadic CreutzfeldtJakob disease. Nature 352:340-342. https://doi.org/10.1038/35234 $\mathrm{Oa} 0$

46. Pedregrosa F (2011) Scikit-learn: machine learning in python. J Mach Learn Res 12:2825-2830

47. Pocchiari M, Puopolo M, Croes EA, Budka H, Gelpi E, Collins S et al (2004) Predictors of survival in sporadic Creutzfeldt-Jakob disease and other human transmissible spongiform encephalopathies. Brain 127:2348-2359. https://doi.org/10.1093/brain/awh24 9

48. Ritchie ME, Phipson B, Wu D, Hu Y, Law CW, Shi W et al (2015) limma powers differential expression analyses for RNA-sequencing and microarray studies. Nucleic Acids Res 43:e47. https://doi. org/10.1093/nar/gkv007

49. Rudge P, Jaunmuktane Z, Hyare H, Ellis M, Koltzenburg M, Collinge J et al (2019) Early neurophysiological biomarkers and spinal cord pathology in inherited prion disease. Brain 142:760 770. https://doi.org/10.1093/brain/awy358

50. Saghafinia S, Mina M, Riggi N, Hanahan D, Ciriello G (2018) Pan-cancer landscape of aberrant DNA methylation across human tumors. Cell Rep 25(1066-1080):e1068. https://doi.org/10.1016/j. celrep.2018.09.082

51. Sanchez-Mut JV, Heyn H, Vidal E, Moran S, Sayols S, DelgadoMorales R et al (2016) Human DNA methylomes of neurodegenerative diseases show common epigenomic patterns. Transl Psychiatry 6:e718. https://doi.org/10.1038/tp.2015.214

52. Schroder K, Zhou R, Tschopp J (2010) The NLRP3 inflammasome: a sensor for metabolic danger? Science 327:296-300. https ://doi.org/10.1126/science.1184003

53. Stocki P, Sawicki M, Mays CE, Hong SJ, Chapman DC, Westaway D et al (2016) Inhibition of the FKBP family of peptidyl 
prolyl isomerases induces abortive translocation and degradation of the cellular prion protein. Mol Biol Cell 27:757-767. https:// doi.org/10.1091/mbc.E15-10-0729

54. Stojnic R, Diez D (2019) PWMEnrich: PWM enrichment analysis. $\mathrm{R}$ package version 4.23.0.

55. Team TBD (2018) BSgenome.Hsapiens.UCSC.hg19: Full genome sequences for Homo sapiens (UCSC version hg19, based on GRCh37.p13). R package version 1.4.0.

56. Terry C, Wenborn A, Gros N, Sells J, Joiner S, Hosszu LL et al (2016) Ex vivo mammalian prions are formed of paired double helical prion protein fibrils. Open Biol. https://doi.org/10.1098/ rsob. 160035

57. Teschendorff AE, Marabita F, Lechner M, Bartlett T, Tegner J, Gomez-Cabrero D et al (2013) A beta-mixture quantile normalization method for correcting probe design bias in Illumina Infinium 450 k DNA methylation data. Bioinformatics 29:189-196. https ://doi.org/10.1093/bioinformatics/bts680

58. Thompson AGB, Mead SH (2019) Review: Fluid biomarkers in the human prion diseases. Mol Cell Neurosci 97:81-92. https:// doi.org/10.1016/j.men.2018.12.003

59. Thompson AG, Lowe J, Fox Z, Lukic A, Porter MC, Ford L et al (2013) The Medical Research Council prion disease rating scale: a new outcome measure for prion disease therapeutic trials developed and validated using systematic observational studies. Brain 136:1116-1127. https://doi.org/10.1093/brain/awt048

60. Turner SD (2018) qqman: an R package for visualizing GWAS results using Q-Q and manhattan plots. biorXiv. https://doi. org/10.1101/005165
61. van Iterson M, van Zwet EW, Consortium B, Heijmans BT (2017) Controlling bias and inflation in epigenome- and transcriptomewide association studies using the empirical null distribution. Genome Biol 18:19. https://doi.org/10.1186/s13059-016-1131-9

62. Zannas AS, Wiechmann T, Gassen NC, Binder EB (2016) Genestress-epigenetic regulation of FKBP5: clinical and translational implications. Neuropsychopharmacology 41:261-274. https://doi. org/10.1038/npp.2015.235

63. Zannas AS, Balsevich G, Gassen NC (2016) The emerging role of FKBP5 in the regulation of metabolism and body weight. Surg Obes Relat Dis 12:1560-1561. https://doi.org/10.1016/j.soard .2016.05.016

64. Zannas AS, Jia M, Hafner K, Baumert J, Wiechmann T, Pape JC et al (2019) Epigenetic upregulation of FKBP5 by aging and stress contributes to NF-kappaB-driven inflammation and cardiovascular risk. Proc Natl Acad Sci USA 116:11370-11379. https://doi. org/10.1073/pnas.1816847116

65. Zhang M, McKeever PM, Xi Z, Moreno D, Sato C, Bergsma T et al (2020) DNA methylation age acceleration is associated with ALS age of onset and survival. Acta Neuropathol 139:943-946. https://doi.org/10.1007/s00401-020-02131-z

Publisher's Note Springer Nature remains neutral with regard to jurisdictional claims in published maps and institutional affiliations. 